\title{
Exame parasitológico de fezes de gatos (Felis catus domesticus) domiciliados e errantes da Região Metropolitana do Rio de Janeiro, Brasil
}

\author{
Parasitological study with faecal samples of stray and domiciliated \\ cats (Felis catus domesticus) from the Metropolitan \\ Area of Rio de Janeiro, Brazil
}

Cathia Maria Barrientos Serra', Claudia Maria Antunes Uchôa²

e Rafaela Alonso Coimbra ${ }^{1}$

\begin{abstract}
Resumo Foram estudadas 131 amostras de fezes de gatos de comportamento domiciliado e errante da Região Metropolitana do Rio de Janeiro, obtendo-se uma positividade de $63,4 \%$ das amostras, com maior ocorrência no grupo de animais errantes. Foi observado predomínio de parasitismo por Ancylostoma sp (43,5\%), Toxocara sp $(19,1 \%)$ e Cystoisospora sp $(43,5 \%)$ em ambos os grupos. Também foram encontrados ovos de Uncinaria sp (1,5\%), Toxascaris leonina (7,6\%), cistos de Giardia sp (6,1\%) e esporocistos de Sarcocystis sp $(0,8 \%)$. A alta prevalência de enteroparasitas na amostra estudada ressalta a importância de um maior controle parasitológico nesses animais, para proteção da saúde animal e humana.
\end{abstract}

Palavras-chaves: Gatos. Parasitismo intestinal. Helmintos. Protozoários. Zoonose.

Abstract In a survey of 131 faecal samples from Rio de Janeiro domiciliated and stray cats, 63,4\% were positive with a higher prevalence among stray animals. Ancylostoma sp (43.5\%), Toxocara sp (19.1\%), Cystoisospora sp (43.5\%), Uncinaria sp (1.5\%), Toxocaris leonine (7.6\%), Giardia sp (6.1\%) and Sarcocystis $\mathrm{sp}(0.8 \%)$ were found. This high prevalence suggests the need for improved control measures against cats to protect animal and human health.

Key-words: Cats. Intestinal parasitism. Helminth. Protozoan. Zoonosis.

Os parasitas intestinais dos animais domésticos de estimação, além de serem responsáveis diretamente por danos à saúde de seus hospedeiros habituais podem, ocasionalmente, infectar o homem, sendo também neste, capazes de acarretar doença.

Os trabalhos sobre a freqüência do parasitismo intestinal em gatos no Brasil ainda são escassos ${ }^{1291016}$, uma vez que a grande maioria concentra-se na avaliação de cães, sendo esta mesma situação observada na Região Metropolitana do Rio de Janeiro, nos últimos anos.

O recente aumento da aquisição de gatos como animais de estimação e os conhecimentos atuais sobre as zoonoses que eles podem transmitir têm estimulado os estudos nesses animais.

O presente trabalho teve como objetivo avaliar a ocorrência de enteroparasitas de gatos de comportamento domiciliado e errante, provenientes da Região Metropolitana do Rio de Janeiro.

\footnotetext{
1. Departamento de Patologia e Clínica Veterinária da Faculdade de Veterinária da Universidade Federal Fluminense, Niterói, RJ. 2. Departamento de Microbiologia e Parasitologia do Instituto Biomédico da Universidade Federal Fluminense, Niterói, RJ.

Endereço para correspondencia: Dra. Cathia M. B. Serra. Disciplina de Zoonoses. DPCV/FMV/UFF. Rua Vital Brazil Filho 64, Santa Rosa, $24230-340$ Niterói, RJ. Tel: 21 2714-8454 Fax: 2126106657

e-mail:uchoa@radnet.com.br

Recebido para publicação em 26/11/1999

Aceito em 8/5/2003
} 


\section{MATERIAL E MÉTODOS}

Foram estudadas 131 amostras fecais de gatos de idades variadas e ambos os sexos procedentes, principalmente, dos bairros da zona norte e sul das cidades de Niterói e Rio de Janeiro, os quais foram divididos em dois grupos a partir de seu comportamento: domiciliado (Grupo I) ou errante (Grupo II). Os animais do grupo I viviam em casas ou apartamentos com seus donos e os do grupo II foram apreendidos em logradouros públicos.

As amostras foram processadas pela técnica de centrifugo-flutuação em solução saturada de açúcar ${ }^{6}$ (densidade 1.150), sendo realizada a leitura de uma lâmina por amostra, em microscópio óptico.

\section{RESULTADOS}

Das 131 amostras fecais de gatos estudadas (65 do Grupo I e 66 do grupo II), 83 (63,4\%) foram positivas (Tabela 1).

Os principais enteroparasitas encontrados nos gatos foram os ancilostomídeos, principalmente Ancylostoma $\mathrm{sp}$, seguido de Cystoisospora sp e pelos ascarídeos com maior freqüência de Toxocara sp (Tabela 2).

Nos gatos domiciliados o monoparasitismo foi observado em 19 (29,2\%) animais. A associação de dois parasitas foi evidenciada em $5(7,7 \%)$ e de três em $1(1,5 \%)$. Nesses gatos o monoparasitismo mais freqüente foi devido a Ancylostoma sp em 16,9\% (11/65) das amostras. O parasitismo múltiplo foi encontrado em $9,2 \%$ $(6 / 65)$, sendo que as associações parasitárias mais comuns foram Ancylostoma sp com Toxocara sp em 3 animais e Ancylostoma sp e Cystoisospora felis em 2.

Nas 66 amostras de animais errantes, o monoparasitismo foi observado em 21/66 (31,8\%) e o parasitismo múltiplo observado em $37 / 66(56,1 \%)$. O monoparasitismo mais freqüente foi devido a Ancylostoma sp em 11/66 amostras. As associações parasitárias mais freqüentes foram entre Ancylostoma sp e Cystoisospora sp em 13/66 amostras e Ancylostoma sp, Toxocara sp e Cystoisospora sp em 5/66 amostras.

\begin{tabular}{|c|c|c|c|c|c|c|}
\hline \multirow[b]{3}{*}{ Amostras } & \multicolumn{2}{|c|}{ Grupo I } & \multicolumn{2}{|c|}{ Grupo II } & \multirow{2}{*}{\multicolumn{2}{|c|}{ Total }} \\
\hline & gatos & domiciliados & Gatos & errantes & & \\
\hline & $\mathrm{n}=$ amostras & $\%$ & $\mathrm{n}^{\circ}$ amostras & $\%$ & $\mathrm{n} \cong$ amostras & $\%$ \\
\hline Positivas & 25 & 38,5 & 58 & 87,9 & 83 & 63,4 \\
\hline Negativas & 40 & 61,5 & 8 & 12,1 & 48 & 36,6 \\
\hline Total & 65 & 49,6 & 66 & 50,4 & 131 & 100,0 \\
\hline
\end{tabular}

Tabela 2 - Prevalência de espécies de enteroparasitas, através do exame coprológico, em amostras fecais de 65 gatos domiciliados e 66 errantes provenientes da Região Metropolitana do Rio de Janeiro.

\begin{tabular}{|c|c|c|c|c|c|c|}
\hline \multirow[b]{3}{*}{ Parasitas } & \multicolumn{2}{|c|}{ Grupo I } & \multicolumn{2}{|c|}{ Grupo II } & & \\
\hline & gatos & domiciliados & Gatos & errantes & \multicolumn{2}{|c|}{ Total } \\
\hline & $\mathrm{n}^{\circ}$ & $\%$ & $\mathrm{n}^{0}$ & $\%$ & $\mathrm{n}^{\circ}$ & $\%$ \\
\hline Ancylostoma sp & 17 & 26,1 & 40 & 60,6 & 57 & 43,5 \\
\hline Uncinaria sp & 0 & 0 & 2 & 3,0 & 2 & 1,5 \\
\hline Toxocarasp & 6 & 9,2 & 19 & 28,8 & 25 & 19,1 \\
\hline Toxascaris leonina & 0 & 0 & 10 & 15,2 & 10 & 7,6 \\
\hline Cystoisospora sp & 8 & 12,3 & 49 & 74,2 & 57 & 43,5 \\
\hline Sarcocystis sp & 1 & 1,5 & 0 & 0 & 1 & 0,8 \\
\hline Giardia sp & 0 & 0 & 8 & 12,1 & 8 & 6,1 \\
\hline
\end{tabular}

\section{DISCUSSÃO}

A alta (87,9\%) prevalência de parasitismo encontrada nos gatos de comportamento errante é atribuída ao fato de serem mal nutridos, de não receberam tratamento antiparasitário e viverem mais expostos a infecções, circulando por diversas áreas o que também favorece a disseminação de enteroparasitas.

Em gatos provenientes de apreensão de rua da cidade de São Paulo, Côrtes et $\mathrm{al}^{2}$ constataram, através do exame de fezes, que $22,7 \%$ dos animais estavam parasitados por Ancylostoma sp e que 17,6\% estavam parasitados por Toxocara sp. Quando Ogassawara et al ${ }^{10}$ necropsiaram o mesmo tipo de gato da mesma cidade, observaram que o parasitismo por Ancylostoma braziliense foi de 37\%, por Ancylostoma caninum/ Ancylostoma tubaeforme foi de $25,9 \%$ e por Toxocara cati foi de $53,7 \%$. Nas amostras de gatos errantes do 
presente trabalho verificou-se, através do exame de fezes, uma alta freqüência tanto para Ancylostoma sp (40/66) como para Toxocara sp (19/66), concordando com o achado desses autores, o que demonstra uma grande dispersão dos mesmos representando risco para a saúde humana podendo causar a larva migrans cutânea $^{13}$, larva migrans visceral ${ }^{11}$ e enterite eosinofílica ${ }^{12} 14$.

Também no grupo I, a ocorrência de ovos de ancilostomídeos $(26,1 \%)$ e ascarídeos $(9,2 \%)$, apesar de não ter sido tão alta como no grupo de gatos errantes, não deve ser menosprezada, pois alguns desses moravam em casas com quintal e não estavam totalmente confinados.

A diversidade dos parasitas encontrados entre os dois grupos foi praticamente a mesma, prevalecendo em ambos os casos as infecções por ancilostomídeos, Toxocara sp, Cystoisospora sp. Em outros estudos ${ }^{15} 310$, realizados através de exame coprológico, estes parasitas foram os mais observados.

O parasitismo múltiplo foi observado em $32,8 \%$ (43/ 131) das amostras, sendo predominante no grupo II (37/ 131). Este resultado foi superior ao observado por Ogassawara et al $^{9}$ que num estudo em São Paulo com 215 gatos de atendimento ambulatorial, domiciliados porém não totalmente confinados, encontraram parasitismo múltiplo em 21,9\%.

Não foi possível observar oocistos tipo ToxoplasmaHammondia em nenhuma amostra estudada, o que não diverge dos dados da literatura, que relata dificuldade no encontro desses oocistos ${ }^{3}$.

O encontro de Uncinaria sp só foi possível em 3\% (2/66) das amostras de gatos errantes. Uchôa et al ${ }^{15}$ alertam sobre a importância na identificação dos ovos deste parasita, já que os mesmos podem ser confundidos com ovos de outros ancilostomídeos, além do fato de ser considerado um parasita de clima frio e incomum no nosso país.

O encontro de Giardia sp foi possível em apenas 8 amostras de animais errantes (12,1\%). Este protozoário tem sido descrito como agente de diarréia nos gatos, principalmente entre os filhotes, sendo seu potencial ainda não esclarecido ${ }^{4}$.

Foram encontrados no Grupo II (animais errante) $15,2 \%$ de amostras com Toxascaris leonina. Este ascarídeo apresenta baixa prevalência em gatos ${ }^{7} 8$, sendo menos patogênico que os outros ascarídeos de cães e gatos ${ }^{11}$. No Brasil, Farias et $\mathrm{al}^{5}$ relataram o encontro deste parasita em $0,9 \%$ (3/314) dos cães estudados.

O encontro de parasitas como Giardia sp, Uncinaria sp e Toxascaris leonina, os quais apresentam pouca citação na literatura brasileira, vem ressaltar a importância da necessidade de um número maior de levantamentos parasitológicos em animais domésticos, e principalmente em gatos, uma vez que tem aumentado o número de proprietários dessa espécie animal devido a sua independência e adaptação a apartamentos.

\section{AGRADECIMENTOS}

À Sociedade Protetora dos Animais (SUIPA) do Rio de Janeiro, ao CCZ-Niterói e a Dra. Alba Valéria Barcelos do Instituto Municipal de Medicina Veterinária Jorge Vaitsman pela coleta do material de gatos errantes.

\section{REFERÊNCIAS BIBLIOGRÁFICAS}

1. Campos DMB, Garibaldi IM, Carneiro JR. Prevalência de helmintos em gatos (Felis catus domesticus) de Goiânia. Revista de Patologia Tropical 3:355-359, 1974.

2. Côrtes VA, Paim GV, Alencar RA. Infestação por ancilostomídeos e toxocarídeos em cães apreendidos em vias públicas, São Paulo (Brasil). Revista de Saúde Publica São Paulo 22:341-343, 1988.

3. Dubey JP. Toxoplasmosis in cats. Feline Practice 16:1226;44-45, 1986.

4. Dubey JP. Intestinal Protozoa Infections. Veterinary Clinical of North America Small Animal Practice 23:37-55. 1993.

5. Farias NA, Christovão ML, Stobbe NS. Frequência de parasitas intestinais em cães (Canis familiaris) e gatos (Felis catus domesticus) em Araçatuba, São Paulo. Revista Brasileira de Parasitologia Veterinária. 4:57-60, 1995.

6. Figueiredo, PC. Infecções naturais por eimerias em bovinos de raças leiteiras no Estado do Rio de Janeiro. Tese de Mestrado. Universidade Federal Rural do Rio de Janeiro. 1982.

7. Guterbock WM, Levine ND. Coccidia and intestinal nematodes of East Central Illinois cats. Journal of American Veterinary Medical Association 170:1411-1413, 1977.
8. Nichol S, Ball SJ, Snow KR. Prevalence of intestinal parasites in domestic cats from London. Veterinary Record 109:252-253, 1981.

9. Ogassawara S, Benassi S, Larsson CE, Hagiwara MK. Prevalência de endoparasitas em gatos na cidade de São Paulo. Revista da Faculdade de Medicina Veterinária e Zootecnia da Universidade de São Paulo 23:39-46, 1986.

10. Ogassawara S, Benassi S, Larsson CE, Leme PTZ, Hagiwara MK. Prevalência de infecções helmínticas em gatos na cidade de São Paulo. Revista da Faculdade de Medicina Veterinária e Zootecnia da Universidade de São Paulo. 23:145-149, 1986.

11. Parsons JC. Ascarid infections of cats and dogs. Veterinary Clinical of North America Small Animal Practice 17:1307-1339, 1987.

12. Provic P, Croese J. Human enteric infection with Ancylostoma caninum: hookworms reappraised in the light of a new zoonosis. Acta Tropica 62:23-44, 1996.

13. Schantz PM. Of worms, dogs, and human hosts: Continuing challenges for veterinarians in prevention of human disease. Journal of American Veterinary Medical Association 204:10231028, 1994.

14. Smyth JD. Rare new emerging helminth zoonoses. Advances in Parasitology 36:1-45, 1995. 
15. Uchôa, CMA, Peixoto, CMS, Mattos Jr. DG, Barcelos AV. Occurrence and identification of Uncinaria Frohlish,1789 (Nematoda: Ancylostomidade) parasites in stray cats (Felis catus) from Rio de Janeiro, Brazil. Revista Brasileira de Parasitologia Veterinária 7:161-164, 1998.
16. Zago Filho H, Barreto MP. Estudo sobre a prevalência e intensidade de infestação por helmintos intestinais em cães e gatos de Ribeirão Preto, São Paulo. Revista Brasileira de Malariologia e Doenças Tropicais 9:295-304, 1957. 